\title{
A MOST probable explanation of the pulsation of WR 123
}

\author{
E. A. Dorfi ${ }^{1}$, A. Gautschy ${ }^{2}$, and H. Saio ${ }^{3}$ \\ 1 Institut für Astronomie der Universität Wien, Türkenschanzstr. 17, 1180 Wien, Austria \\ e-mail: dorfi@astro.univie.ac.at \\ 2 Wetterchrüzstr. 8c, 4410 Liestal, Switzerland \\ 3 Astronomical Institute, Tohoku University, Sendai, Japan \\ Received 28 November 2005 / Accepted 18 May 2006
}

\section{ABSTRACT}

\begin{abstract}
Aims. Resorting to the observed period of the cyclic part of WR 123's photometric variability we constrain its stellar parameters. Methods. Radiation hydrodynamic simulations were performed on selected stellar envelope models for which also linear stability analyses were computed.

Results. Pulsations of massive hydrogen-rich stellar envelopes subject to sufficiently high luminosity-to-mass ratios are excited with periods that conform with the observed one by MOST in WR123.
\end{abstract}

Key words. stars: massive - stars: pulsations - stars: individual: WR123

\section{Introduction}

Most of Wolf-Rayet (WR) stars are evolved luminous stars characterized by hydrogen-poor envelopes and dense winds (e.g. Abbott \& Conti 1987). Among the WR stars, the subtype WN8 stars are currently thought of being direct evolutionary descendants of massive and luminous Of stars Marchenko et al. (1998) and show various spectroscopic and light variations on a variety of time scales. Some of the variations were suspected to be caused by stellar pulsations, and periodic variations had been searched for in WN8 stars (Marchenko et al. 1998, and references therein). These attempts have been inconclusive until the MOST satellite detected recently a light variation with a period of $9.8 \mathrm{~h}$ in the WN8 star WR123 Lefèvre et al. (2005).

Radial and non-radial pulsations of very luminous stars are excited by strange-mode excitation that works in radiationpressure dominant layers caused by an opacity peak (Gautschy \& Glatzel 1990; Glatzel \& Gautschy 1992; Glatzel 1994; Glatzel \& Mehren 1996; Saio et al. 1998). Using helium-star models for WR stars, Glatzel et al. (1993) showed that pulsations were excited with periods shorter than $30 \mathrm{~min}$. Later, Glatzel et al. (1999) performed nonlinear pulsation analysis for some of those excited modes and found that these pulsations grew to appreciable amplitudes.

Referring to their results, Lefèvre et al. (2005) concluded that the $9.8 \mathrm{~h}$-period they discovered was too long for the pulsation to be excited by strange-mode instability. Contrary to their conclusion, we will show in this letter that the observed period is in fact very consistent with a pulsation excited by strange-mode instability in models for WR 123.

The discrepancy comes from the fact that Lefèvre et al. (2005) did not take into account the difference in stellar radius $\left(R_{*}\right)$ between WR 123 and helium star models of Glatzel et al. (1999). The stellar radius estimated by Crowther (1997) is about $15.3 R_{\odot}$ with very low hydrogen and Hamann \& Koesterke (2000) give $18.9 R_{\odot}$, while the models of the helium stars (Glatzel et al. 1999) are similar to or less than $1 R_{\odot}$. Since the pulsation period of a chosen mode order is roughly proportional to $R_{*}^{3 / 2}$, the periods of the unstable modes of helium stars in Glatzel et al. (1999) shift into a range which is compatible with the observed period of WR 123.

\section{Modeling WR123's pulsation}

Hydrostatic hydrogen-rich stellar envelope models were computed for a range of stellar masses and luminosities. Most of the models were assumed to have $X=0.35$ and $Z=0.02$, an abundance choice which seems to be in accordance with Hamann \& Koesterke (1998) for average WN8-type stars. The spatial integrations of the envelopes extended to a temperature of $10^{7} \mathrm{~K}$. On these envelope models, linear non-adiabatic stability analyses were computed using the method described in Gautschy \& Glatzel (1990). Periods and growth rates of the lowest few radial modes were computed. Nonlinear radiation hydrodynamic (RHD) simulations were performed for the particularly well suited model parameters that led to linear periods close to the observed one in WR 123. Linear analysis with lower values of $X=0.1$ show that the periods shift but the instability found remains rather robust and is caused by the "Fe-bump".

The numerical method underlying the RHD code has been outlined in detail by Dorfi (1998). We only briefly mention three important technical features. First, the most accurate numerical results are obtained in dynamically changing stellar envelopes when using an adaptive grid which concentrates the moving grid points at steep gradients as well as propagating fronts. Secondly, the temporal discretization relies on time-centered variables allowing second order accuracy in time. Thirdly, the physical quantities are transported across the cell boundaries with the help of a second-order monotonic advection scheme. We emphasize that all three of these numerical features are essential to perform RHD simulations on pulsating stellar envelopes. Typically about 100 time steps are needed to compute a whole pulsational cycle.

The initially hydrostatic models are taken from standard stellar evolution calculations and adopted for the non-linear RHD computations by integrating them inwards down to pressure 
Table 1. Collection of those WR123 models with periods around 0.5 days. The first five columns show the values of the initial hydrostatic models. Note that $T_{\text {eff }}$ and $R_{\mathrm{p}}$ refer to model structures devoid of a wind. Starting with the sixth column, results of the non-linear computations are given such as the period $P$ in days, the relative changes of the photospheric radius $R_{\mathrm{p}}$, the velocity difference at the photosphere in km s${ }^{-1}$; the last column lists the bolometric amplitudes in magnitudes as achieved in the limit cycles.

\begin{tabular}{|c|c|c|c|c|c|c|c|c|}
\hline Name & $\begin{array}{c}M \\
{\left[M_{\odot}\right]}\end{array}$ & $\begin{array}{c}L \\
{\left[10^{5} L_{\odot}\right]}\end{array}$ & $\begin{array}{l}T_{\text {eff }} \\
{[\mathrm{K}]}\end{array}$ & $\begin{array}{c}R_{\mathrm{p}} \\
{\left[R_{\odot}\right]}\end{array}$ & $\begin{array}{c}P \\
\text { [days] }\end{array}$ & $\Delta R_{\mathrm{p}} / R_{\mathrm{p}}$ & $\begin{array}{c}\Delta u \\
{\left[\mathrm{~km} \mathrm{~s}^{-1}\right]}\end{array}$ & $\begin{array}{l}\Delta m_{\text {bol }} \\
{[\mathrm{mag}]}\end{array}$ \\
\hline WR123B & 22 & 3.16 & 39000 & 12.31 & 0.482 & 0.29 & 386 & 0.51 \\
\hline WR123D & 23 & 3.16 & 38000 & 12.97 & 0.499 & 0.22 & 385 & 0.50 \\
\hline WR123F & 23 & 3.16 & 40000 & 11.70 & 0.423 & 0.22 & 408 & 0.54 \\
\hline WR123G & 22 & 3.16 & 40000 & 11.70 & 0.446 & 0.23 & 403 & 0.54 \\
\hline WR123H & 25 & 3.16 & 38000 & 12.97 & 0.427 & 0.19 & 425 & 0.72 \\
\hline WR123L & 27 & 3.16 & 38000 & 12.97 & 0.344 & 0.02 & 58 & 0.10 \\
\hline WR123M & 26 & 3.16 & 38000 & 12.97 & 0.391 & 0.07 & 158 & 0.27 \\
\hline WR123N & 25 & 3.00 & 38000 & 12.63 & 0.377 & 0.10 & 288 & 0.46 \\
\hline WR123P & 23 & 3.00 & 38000 & 12.63 & 0.457 & 0.21 & 406 & 0.61 \\
\hline WR123U & 25 & 2.82 & 33900 & 15.38 & 0.493 & 0.10 & 261 & 0.39 \\
\hline
\end{tabular}

value of $\log P=13$ (in cgs units) where the radial pulsational amplitude is zero. All models given in Table 1 were perturbed by a spatial polynomial that reaches an amplitude of $5 \mathrm{~km} \mathrm{~s}^{-1}$ at the outer boundary. The initial boundary-value problem was integrated into the nonlinear saturation episode in all cases. For more detailed comparisons with observations, we computed light curves in various photometric passbands by performing a non-grey static radiative transfer at each time level of the simulations. Using the computed temperature, density, and pressure stratification we obtained light curves in the standard $B$ and $V$ passbands. The radiative transfer was calculated at the 1212 frequency points prescribed by Kurucz's monochromatic opacities (Kurucz 1993). The resulting spectral data were convolved with standard photometric filter curves to obtain the corresponding synthetic light curves (more details can be found in Dorfi \& Gautschy 2000).

\section{Results}

The stellar parameters of our model stars were chosen to be qualitatively compatible with the contents of Fig. 4 in Hamann \& Koesterke (2000) which shows the distribution of their WN-type stars on the HR plane. Those model stars with stellar parameters leading to at least one overstable pulsation mode around 0.5 days were then selected for non-linear RHD simulation runs. Table 1 contains, for all WR 123-relevant models, the basic stellar parameters and some nonlinear pulsation properties. Comparing the periods (see sixth column in Table 1) we see that a number of WN models may be compatible with the MOST observations of WR 123.

In the following, we pick out model WR123U from Table 1 to present the linear and nonlinear pulsation behaviour; it is representative for all other models.

Figure 1 displays the spatial variation of linear non-adiabatic pulsation quantities: the relative radial displacement $\delta r / r$ and the differential work $\mathrm{d} W / \mathrm{d} r$ ( $>0$ for excitation). The latter quantity is arbitrarily normalized to its maximum value. For further elucidation, the scaled spatial variation of $\kappa_{T} \equiv(\partial \log \kappa / \partial \log T)_{P}$ is plotted throughout the stellar envelope whose depth is measured in $\log T$. Evidently, the displayed fundamental mode, with a period of 0.406 days, is excited in the range $5.0<\log T<5.3$; according to $\kappa_{T}$ this corresponds to the domain of the "Fe-bump". From $\delta r / r$ we see that in radius about the outermost $10 \%$ of the star participates in the pulsation; this region engulfs, however, only about $5 \times 10^{-7} M_{*}$. Hence, the pulsating layers are very tenuous and embedded in a very strong radiation field.

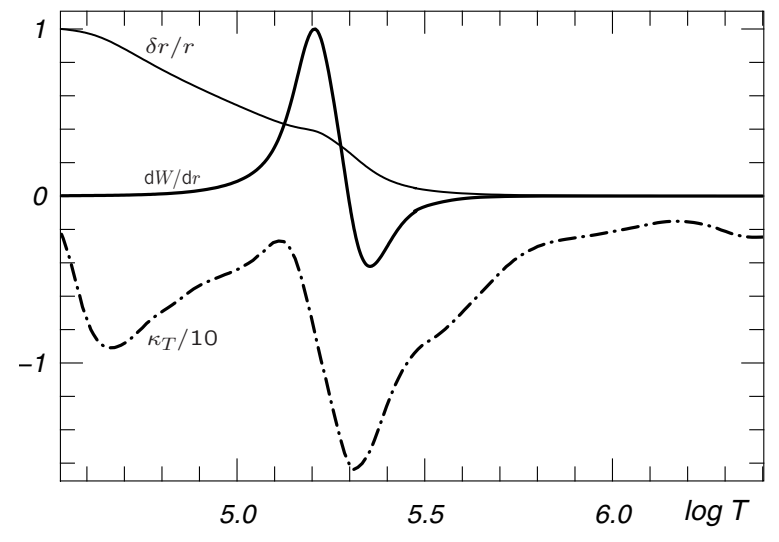

Fig. 1. Spatial variation of selected physical quantities for the representative model WR123U. The curves labelled $\delta r / r$ and $\mathrm{d} W / \mathrm{d} r$ stand for the relative radius perturbations and the differential work, respectively. The dash-dotted curve delineates the scaled spatial variation of the opacity derivative $\kappa_{T} \equiv(\partial \log \kappa / \partial \log T)_{P}$.

Characteristic of strange-mode - type instabilities, the perturbations are strongly confined; in this case to the outermost regions and bounded in the interior by the "Fe-bump".

Due to the models' high luminosities, radiation pressure dominates the atmospheric stratification, in particular for the initial hydrostatic models. As the pulsation develops the atmospheric layers usually are levitated and, depending on the amplitude of the pulsations, the outermost layers can expand. In the case of WR123U, the pulsation occurs around a mean photospheric radius of $\bar{R}_{\mathrm{p}}=15.71 R_{\odot}$ at a lower mean temperature of $\bar{T}=33540 \mathrm{~K}$ compared to an initial effective temperature at $T_{\text {eff }}=30900 \mathrm{~K}$. Hence, we expect the non-linear period of $P=0.493$ days to be longer than the linear period as computed on the hydrostatic stellar structure. All the models exhibit largescale motions within the stellar atmospheres where the outermost layers are often not synchronized with the internal stellar pulsations (more details are presented in Dorfi \& Gautschy 2006).

The temporal evolution of the pulsational velocity (in $\mathrm{km} \mathrm{s}^{-1}$ ) of model $\mathrm{W} 123 \mathrm{U}$ is depicted in Fig. 2 over the first 30 days after the initial perturbation. Typical for such strangemode - like instabilities is their fast growth time; the dotted line represents the amplitude evolution predicted by the linear nonadiabatic analysis which gave an e-folding time of 11.5 days. Already after about 28 days the final velocity amplitude was 


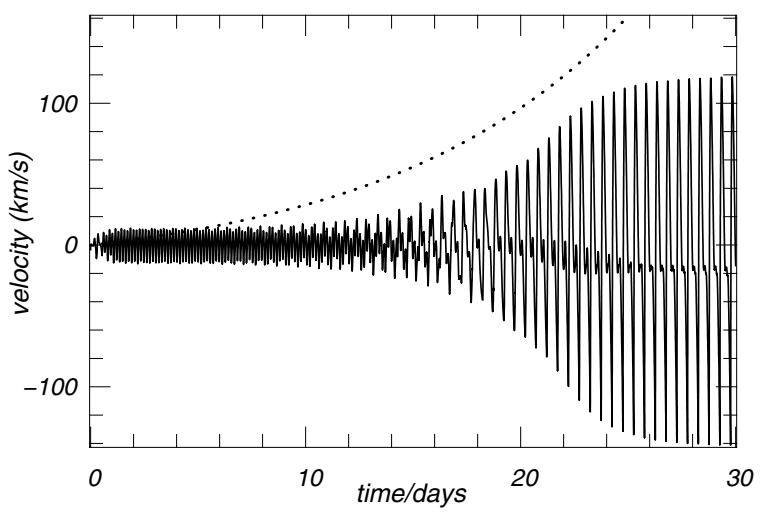

Fig. 2. Initial growth of photospheric velocity of model WR123U. The dotted line indicates the growth of the amplitude as computed from a linear non-adiabatic analysis. After about 28 days, non-linear saturation forces the model into its final pulsation amplitude.

reached ranging from $+120 \mathrm{~km} \mathrm{~s}^{-1}$ to $-140 \mathrm{~km} \mathrm{~s}^{-1}$ at the level of the photosphere. The thickness of the atmosphere, i.e. the spatial difference between the photosphere and the outer boundary (moving in a Lagrangian way), varied between 0.006 and 0.058 in units of the mean photospheric radius of $\bar{R}_{\mathrm{p}}=15.71 R_{\odot}$. We emphasize this because the material shells outside the photosphere develop strong shock waves which affect the resulting light curve.

Figure 3 plots synthetic $B$ - and $V$-light curves of model WR123U over phase covering two periods. The small-scale amplitude features are due to the motions induced in the outermost layers and will be even more pronounced in real objects which are not constrained by the assumption of pure radial symmetry. The symmetry between the ascending and descending part of the light curve is due to different spatial extension of the atmosphere at different pulsational phases: Due to the compressibility of the stellar material, the outer layers are almost simultaneously driven outwards by the driving region but later the different layers fall back on their own characteristic dynamical trajectories. The shoulder in the light curve seen around phase 0.8 is caused by outer layers colliding with more slowly inward-moving matter and thereby compressing the whole atmosphere. The synthetic $B$ - and $V$-amplitudes reach $0.18 \mathrm{mag}$ and $0.17 \mathrm{mag}$, respectively. These values are about four times as large as what MOST observes in WR123 (Lefèvre et al. 2005) during the $9.8 \mathrm{~h}$ period. An asymmetric stellar wind can, however, lower the observable amplitude of such luminosity variations (see Dorfi \& Gautschy 2006). As seen in Table 1, the WR123 models WR123M or WR123L with their slightly larger masses also yield smaller amplitudes.

\section{Conclusions}

In contrast to the conclusions in Lefèvre et al. (2005), we find it easy to arrive at pulsating model stars in the range of 22 to $27 M_{\odot}$ with variability periods of the order of half a day. Our models are, however, hydrogen-rich $(X=0.35, Z=0.02)$ and are not of the more compact helium-main-sequence-type as referred to in the conclusions in Lefèvre et al. (2005). A large number of linear stability analyses and ensuing non-linear RHD-computations (some of them are summarized in Table 1) confirm this finding. Our results are stable with respect to a change of chemical composition. Upon going from $X=0.35, Z=0.02$ to $X=0.5, Z=0.02$, the growth rates computed in the linear analyses remain essentially unchanged; in particular, the same modes

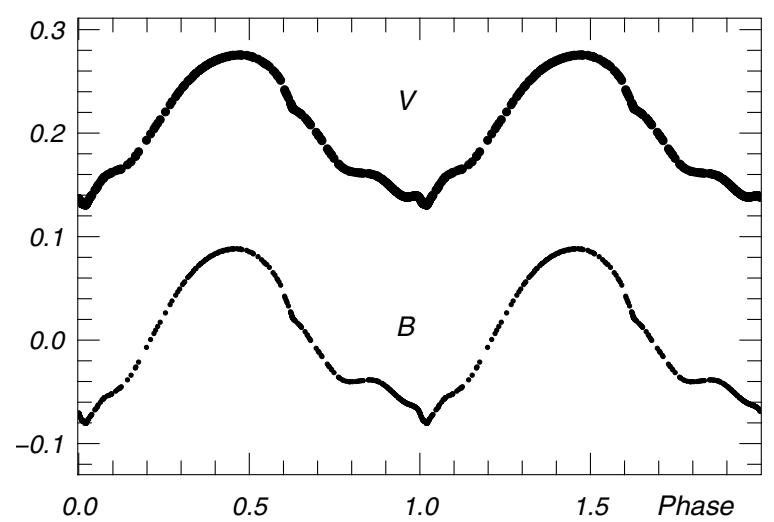

Fig. 3. Light curves in the $B$ and $V$ filter passbands as a function of pulsational phase for the representative model WR123U. The period of the variability is $0.493 \mathrm{~d}$. The $V$ light curve is shifted arbitrarily by 0.2 mag.

remain unstable or stable. This is not further surprising as the "Fe-bump" is responsible for the destabilisation of the modes.

The results of these first, admittedly oversimplified, radiation-hydrodynamic simulations show furthermore that the light curves and the nonlinear amplitudes are compatible with the observed cyclic behaviour of WR123. Hence, we conclude that the regular part of the WR123 variability can be attributed to a strange-mode - like instability. To model a more naturalistic behaviour, the interaction of the pulsating envelope with a hot, dense wind which eventually determines the observables will be unavoidable but also considerably more complicated. Non-linear RHD-computations for LBVs as encountered in the Galactic center demonstrate that similar strange-mode pulsations persist also in envelopes feeding pulsation-driven stellar winds which will be discussed in a forthcoming paper (Dorfi \& Gautschy 2006). Hence, strange-mode - like pulsations are not confined to hydrostatic structures. We are confident that the current models capture the essentials of what drives the cyclic variability of WR 123 inside the sonic point and further variability can probably be attributed to the time-dependent wind zone. Furthermore, we conjecture that this type of variability is common in WN-type stars with sufficiently high luminosity-to-mass ratios.

Acknowledgements. We would like to thank the referee T. Moffat for his valuable comments on the manuscript. This work has not been supported by any other national science funding.

\section{References}

Crowther, P. A., Hilliar, D. J., \& Smith, L. J. 1995, A\&A, 293, 403 Abbott, D., \& Conti, P. S. 1987, ARA\&A, 25, 113

Crowther, P. A. 1997, in Luminous Blue Variables: Massive Stars in Transition, ed. A. Nota, \& H. J. G. L. M. Lamers (ASP), 120, 51

Dorfi, E. A. 1998, 27th Saas Fee Course (Berlin: Springer), 263

Dorfi, E. A., \& Gautschy, A. 2000, ApJ, 545, 982

Dorfi, E. A., \& Gautschy, A. 2006, A\&A, in preparation

Gautschy, A., \& Glatzel, W. 1990, MNRAS, 245, 597

Glatzel, W. 1994, MNRAS, 271, 66

Glatzel, W., \& Gautschy, A. 1992, MNRAS, 256, 209

Glatzel, W., Kiriakidis, M., \& Fricke, K. J. 1993, MNRAS, 262, L7

Glatzel, W., Kiriakidis, M., Chernigovskij, S., \& Fricke, K. J. 1999, MNRAS, 303,116

Glatzel, W., \& Mehren, S. 1996, MNRAS, 282, 1470

Hamann, W.-R., \& Koesterke, L. 1998, A\&A, 335, 1003

Hamann, W.-R., \& Koesterke, L. 2000, A\&A, 360, 647

Kurucz, R. L. 1993, CDROM 13: ATLAS9.SAO, Harvard, Cambridge

Lefèvre, L., Marchenko, S. V., Moffat, A. F. J., et al. 2005, ApJ, 634, L109

Marchenko, S. V., Moffat, A. F. J., Eversberg, T., et al. 1998, MNRAS, 294, 642

Saio, H., Baker, N. H., \& Gautschy, A. 1998, MNRAS, 294, 622 